\title{
Direct Observations of Structural Phase Transitions in Planar Crystallized Ion Plasmas
}

\author{
T. B. Mitchell,* J. J. Bollinger, D. H. E. Dubin, X.-P. Huang, \\ W. M. Itano, R. H. Baughman
}

\begin{abstract}
Laser-cooled ${ }^{9} \mathrm{Be}^{+}$ions confined in two-dimensionally extended lattice planes were directly observed, and the images were used to characterize the structural phases of the ions. Five different stable crystalline phases were observed, and the energetically favored structure could be sensitively tuned by changing the areal density of the confined ions. The experimental results are in good agreement with theoretical predictions for the planar (infinite in two dimensions) one-component plasma. Qualitatively similar structural phase transitions occur, or are predicted to occur, in other experimentally realizable planar systems.
\end{abstract}

The one-component plasma (OCP) has been a model of condensed matter in statistical physics for over 30 years, and it is used to describe such diverse systems as dense astrophysical matter (1) and electrons on the surface of liquid helium (2). Laser-cooled trapped ions (3) are an excellent experimental realization of the OCP. The phase structures of spatially homogeneous (infinite) (4) and cylindrical (infinite in one dimension only) (5) OCPs have been explored previously. Here, images of individual ions that were confined in twodimensionally extended lattice planes are presented and used to characterize the structural phases; the observed structures agree well with the predictions of an analytic theory for the planar OCP.

The OCP model consists of a single charged species embedded in a uniform, neutralizing background charge. In Paul (6) or Penning $(6,7)$ traps, which are used to confine charged particles, a (fictitious) neutralizing background is provided by the confining potentials. The thermodynamic properties of the infinite classical OCP are determined by its Coulomb coupling parameter, $\Gamma \equiv$ $\left[1 /\left(4 \pi \epsilon_{0}\right)\right]\left[e^{2} /\left(a_{\mathrm{ws}} k_{\mathrm{B}} T\right)\right]$, which is the ratio of the Coulomb potential energy of neighboring ions to the kinetic energy per ion; $\epsilon_{0}$ is the permittivity of the vacuum, $e$ is the charge of an ion, $k_{\mathrm{B}}$ is Boltzmann's constant, $T$ is the temperature, and $a_{\mathrm{ws}}$ is the Wigner-Seitz radius [defined by $4 \pi\left(a_{\mathrm{ws}}\right)^{3 / 3}=1 / n_{0}$, where $n_{0}$ is the ion density]. The onset of shortrange order for the infinite OCP is predicted (8) at $\Gamma \approx 2$, and a phase transition to a

T. B. Mitchell, J. J. Bollinger, X.-P. Huang, W. M. Itano, Time and Frequency Division, National Institute of Standards and Technology, Boulder, CO 80303, USA. D. H. E. Dubin, Department of Physics, University of California at San Diego, La Jolla, CA 92093, USA. R. H. Baughman, Research and Technology, Allied Signal, Morristown, NJ 07962, USA.

*To whom correspondence should be addressed. Email: travis.mitchell@nist.gov body-centered cubic (bcc) lattice is predicted $(8,9)$ at $\Gamma \approx 170$. With an OCP in a planar geometry (infinite in only two dimensions), boundary effects are predicted to cause the formation of a variety of additional structural phases, such as the hexagonal close-packed (hcp) and face-centered cubic (fcc) phases $(10,11)$. Qualitatively similar structural phase transitions occur, or are predicted to occur, in other planar systems with varied interparticle interactions, such as plasma dust crystals (12), colloidal suspensions (13), semiconductor electron bilayer systems (14), and hard spheres (15).

The crystallization of small numbers (total number $N<50$ ) of laser-cooled ions into Coulomb clusters (16) was first observed in Paul traps (17). With larger numbers of trapped ions, concentric shells (18) were directly observed in Penning (19) and Paul traps $(5,20)$. Recently, Bragg diffraction has been used to detect bcc crystals (the predicted infinite volume ordering) in large and spherical $\left(N>2 \times 10^{5}\right.$; radius $\left.r_{0}>60 a_{\mathrm{ws}}\right)$ ion plasmas confined in a Penning trap (4).

Measurements were taken from direct images of the central $(r=0)$ structure of pancake-shaped (lenticular) ion plasmas (aspect ratio $\alpha \equiv z_{0} / r_{0}<0.1$, where $2 z_{0}$ is the plasma center's axial extent). The central region has a disklike geometry with constant areal density $\sigma_{0}$ (charge density per unit area projected onto the $z=0$ plane), which facilitates comparison with planar theory. We observed five different stable crystalline phases and found that the energetically favorable central structure can be tuned by changing $\sigma_{0}$. Both continuous and discontinuous structural phase transitions were observed.

The ${ }^{9} \mathrm{Be}^{+}$ions were confined radially in a cylindrical Penning trap (inner trap diameter, $40.6 \mathrm{~mm}$ ) (Fig. 1) with a uniform magnetic field $B=4.465 \mathrm{~T}$ in the $\hat{\mathbf{z}}$-axis direction. The ions were confined axially by a potential difference of $\mathrm{V}_{0}=-1.50 \mathrm{kV}$, which was applied between the center and end electrodes of the trap. Near the trap center, this axial potential is quadratic and has a value of $1 / 2(m / e) \omega_{z}^{2} z^{2}$, where the axial frequency $\omega_{z} /$ $2 \pi=978 \mathrm{kHz}$ for ${ }^{9} \mathrm{Be}^{+}$. The radial electric fields of the trap, as well as the ion space charge, cause the ion plasma to undergo an $\mathbf{E}$ $\times \mathbf{B}$ drift and thus rotate about the trap axis. In thermal equilibrium, this rotation is at a uniform frequency $\omega_{\mathrm{r}}$. The radial binding force of the trap is determined by the Lorentz force caused by the plasma's rotation through the magnetic field. Thus, low $\omega_{\mathrm{r}}$ results in a weak radial binding and a lenticular plasma with a large radius. For $10^{4}$ trapped ions with $\omega_{\mathrm{r}} / 2 \pi=68.5 \mathrm{kHz}$ (typical for this work), the ion plasma has a density of $2.1 \times 10^{8} \mathrm{~cm}^{-3}$ with $2 r_{0} \approx 1.3 \mathrm{~mm}$ and an aspect ratio $\alpha \approx$ 0.05 . The rotation frequency was controlled by phase-locking the plasma rotation to an applied "rotating wall" electric field (21). At low $\omega_{r}$, an increase in $\omega_{r}$ increases both the plasma density and $z_{0}$, providing a way to sensitively adjust the central areal density of the plasma.

The ions were cooled (3) by a laster beam propagating along the $z$ axis and tuned 10 to $20 \mathrm{MHz}$ lower in frequency than a hyperfineZeeman component of the $2 s{ }^{2} S_{1 / 2} \rightarrow 2 p$ ${ }^{2} P_{3 / 2}$ resonance at $313 \mathrm{~nm}$ with a natural linewidth of $19 \mathrm{MHz}$. The laser power was $\sim 50 \mu \mathrm{W}$ and was focused at the ion plasma to a diameter of $\sim 0.5 \mathrm{~mm}$. The theoretical cooling limit is $0.5 \mathrm{mK}$, and an experimental upper bound of $T<10 \mathrm{mK}$ has been measured (22). For a density of $n_{0}=2 \times 10^{8}$ $\mathrm{cm}^{-3}$, these limits give a range of $160<\Gamma<$ 3150. A series of lenses formed side- and top-view images of the ions, with viewing directions that were perpendicular and parallel to the magnetic field, respectively, on either a gateable charge-coupled device (CCD) camera or on an imaging photomultiplier tube. The resolution of the optical system is $\sim 4 \mu \mathrm{m}$, whereas typical interparticle spacings are $\sim 20 \mu \mathrm{m}$.

The side-view image insert in Fig. 1, which shows the central region of a lenticular ion plasma with three axial lattice planes, is representative of the flatness and radial extent $\left(<10 \%\right.$ of $\left.r_{0}\right)$ of this region. At large radius, curvature of the planes can cause the sideview images of axial plane positions to blur. This effect was prevented in the measurements reported here by using clouds with sufficient amounts (up to 50\%) of nonfluorescing impurity ions. Because these heaviermass ions are centrifugally separated to larger radii than the ${ }^{9} \mathrm{Be}^{+}$, the regions of the plasma where curvature begins to be significant can be filled with these ions, which are sympathetically cooled by the ${ }^{9} \mathrm{Be}^{+}(23)$.

With good alignment of the trap with the magnetic field $\left(<10^{-3} \mathrm{rad}\right)$, the ion plasma 
rotation is phase-locked with the rotating wall perturbation (21). A direct observation of the rotating ion structures was achieved for the first time by gating the top-view CCD camera synchronously with the rotating wall perturbation for brief gate times $(<2 \%)$ of the plasma rotation period). Total exposure times of $\sim 3 \times 10^{4}$ rotation periods were used for typical images (Fig. 2). For our study of the ion lattice structure, we limited our analysis to the central region, where strong localization and regular ordering of the ions were observed. At larger radius, we observed an increased blurring (due to the plasma rotation), occasional lattice distortions, and, ultimately, the transition to the regions filled by heavier mass ions.

The observed structure of the central crystallized region depends on the central areal density $\sigma_{0}$ of the plasma. Within a layer, the lattice is characterized by the primitive vectors $\mathbf{a}_{1}$ and $\mathbf{a}_{2}$ (which were observed to be equal in magnitude, $\left|\mathbf{a}_{1}\right|=\left|\mathbf{a}_{2}\right| \equiv a$ ) or, equivalently, by $a$ and the angle $\theta\left(\leq 90^{\circ}\right)$ between the primitive vectors. The interlayer structure is characterized by the axial positions $z_{n}$ of the $n$ lattice planes (measured by the side-view camera) and the interlayer displacement vector $\mathbf{c}_{\mathrm{n}}$ between layers 1 and $n$. Hence, the equilibrium $(x, y)$ positions of ions in axial planes 1 and $n$ are given by $\mathbf{R}_{1}(i, j)=i \mathbf{a}_{1}+j \mathbf{a}_{2}$ and $\mathbf{R}_{n}(i, j)=i \mathbf{a}_{1}+$ $j \mathbf{a}_{2}+\mathbf{c}_{n}$, where $i$ and $j$ are integers.

Three different types of intralayer ordering were observed: hexagonal $\left(\theta=60^{\circ}\right)$, square $(\theta$ $\left.=90^{\circ}\right)$, and rhombic $\left(90^{\circ}>\theta \geq 65^{\circ}\right)$. The square and rhombic layers stack in a staggered fashion, with the upper ions immediately above the centers of the parallelograms below, resulting in an interlayer displacement vector $\mathbf{c}_{2}=$ $\left(\mathbf{a}_{1}+\mathbf{a}_{2}\right) / 2$. Hexagonal layers also stack with ions above the centers of the triangles below, but this stacking can occur in two distinct ways: $3 \mathbf{c}_{2}=\mathbf{a}_{1}+\mathbf{a}_{2}$ and $3 \mathbf{c}_{2} / 2=\mathbf{a}_{1}+\mathbf{a}_{2}$. With hcp-like stacking, the ions in every other plane lie directly above each other ( $a b a b . .$.$) , where-$ as with fcc-like stacking, the ions in every third plane are so aligned $(a b c a b c \ldots)$. When there were three or more hexagonal layers, both types of stacking were observed.

Fig. 1. Schematic side view of the cylindrical Penning trap with its side- and top-view imaging optics. The insets show the variables used to characterize the intra- and interlayer structure. The side-view inset also shows the central region of a lenticular ion plasma with three axial lattice planes. The rotation frequency of the ion plasma was controlled by applying properly phased sinusoidally time-varying electric potentials to the sixfold azimuthally segmented compensation electrodes.

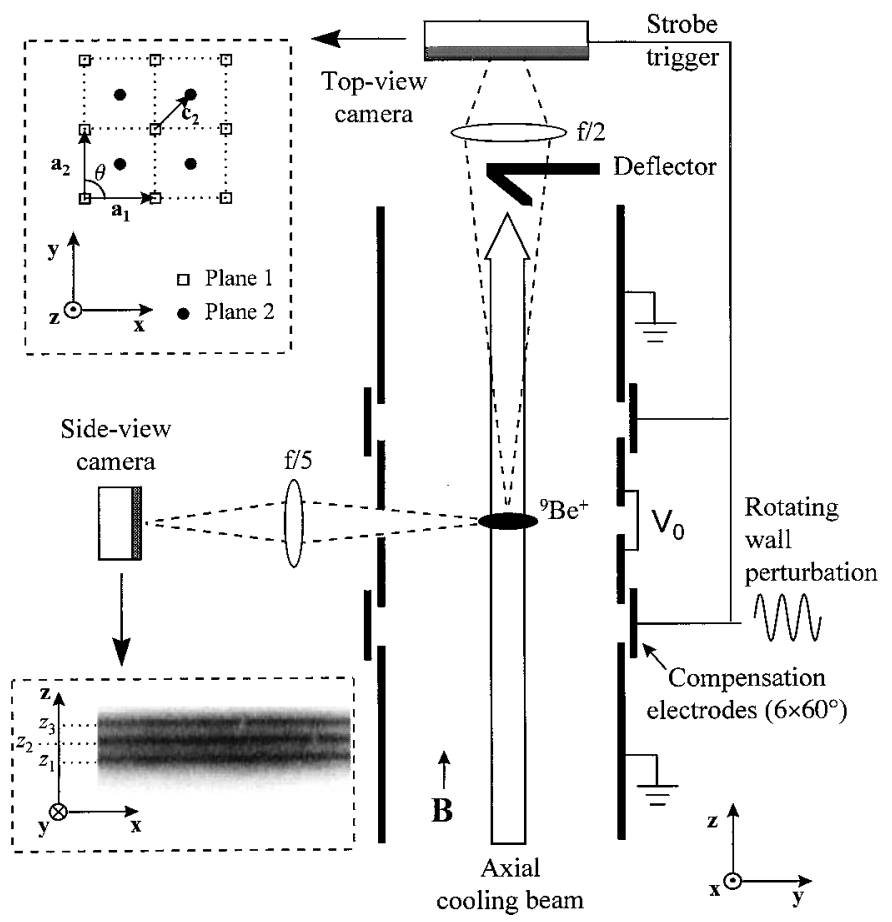

Table 1. Primitive and interlayer displacement vectors in the $(x, y)$ plane for the observed phases. The primitive vector $\mathbf{a}_{1}$ defines the $\hat{\mathbf{x}}$ direction, and $\left|\mathbf{a}_{1}\right|=\left|\mathbf{a}_{2}\right| \equiv a$. Dashes, not applicable.

\begin{tabular}{lllllll}
\hline \multirow{2}{*}{ Phase } & \multirow{2}{*}{ Symmetry } & Stacking & \multicolumn{5}{c}{ Vectors } \\
\cline { 3 - 7 } & & $\mathbf{a}_{1}$ & \multicolumn{1}{c}{$\mathbf{a}_{2}$} & $\mathbf{c}_{2}$ & $\mathbf{c}_{3}$ \\
\hline I & Hexagonal & Single plane & $(a, 0)$ & $\left(a \cos 60^{\circ}, a \sin 60^{\circ}\right)$ & - & - \\
III & Square & Staggered & $(a, 0)$ & $(0, a)$ & $\left(\mathbf{a}_{1}+\mathbf{a}_{2}\right) / 2$ & $(0,0)$ \\
IV & Rhombic & Staggered & $(a, 0)$ & $(a \cos \theta, a \sin \theta)$ & $\left(\mathbf{a}_{1}+\mathbf{a}_{2}\right) / 2$ & $(0,0)$ \\
V & Hexagonal & hcp-like & $(a, 0)$ & $\left(a \cos 60^{\circ}, a \sin 60^{\circ}\right)$ & $\left(\mathbf{a}_{1}+\mathbf{a}_{2}\right) / 3$ & $(0,0)$ \\
$\mathrm{V}_{\text {fcc }}$ & Hexagonal & fcc-like & $(a, 0)$ & $\left(a \cos 60^{\circ}, a \sin 60^{\circ}\right)$ & $\left(\mathbf{a}_{1}+\mathbf{a}_{2}\right) / 3$ & $2\left(\mathbf{a}_{1}+\mathbf{a}_{2}\right) / 3$ \\
\hline
\end{tabular}

The following sequence of phase structures, with lattice parameters defined in Table 1 , were observed as the central areal density $\sigma_{0}$ was increased from where order was first observed: (I) one-layer hexagonal $\rightarrow$ (III) two-layer staggered square $\rightarrow$ (IV) two-layer staggered rhombic $\rightarrow(\mathrm{V})$ two-layer staggered hexagonal. At a critical density, a third layer was formed, resulting in a (III) threelayer staggered square. The process then repeated with minor variations, such as phase III becoming less common. We have followed the classifications used in previous theoretical studies of quantum (14) and classical (11) electron bilayer systems. Phase II, which is a stable phase of the bilayer systems where the interlayer distance is fixed, is not listed here because it is unstable for the planar OCP, where the interlayer distance can vary.

We have performed an analytical calculation of the energies of these phase structures for the planar OCP (24). The calculation minimizes the energy (25) of several parallel lattice planes that are infinite and homogeneous in the $(x, y)$ direction but are confined in the $\hat{\mathbf{z}}$ direction by a harmonic external electrostatic confinement potential

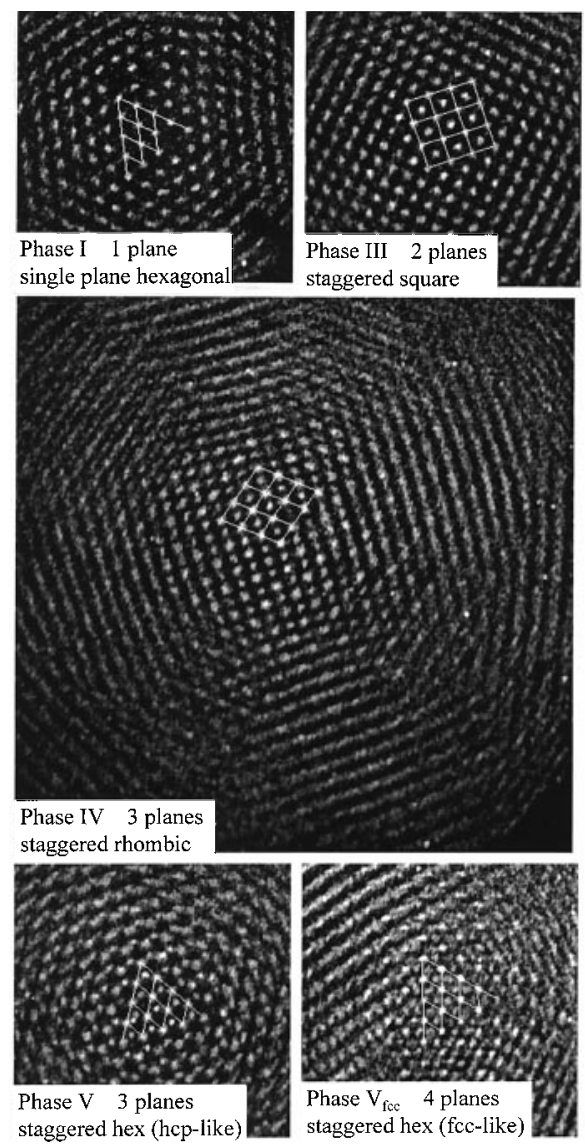

Fig. 2. Top-view $(x, y)$ images of the five structural phases observed in the experiment, with lines showing a fit of the central ions to the indicated structure. 


\section{RE POR T S}

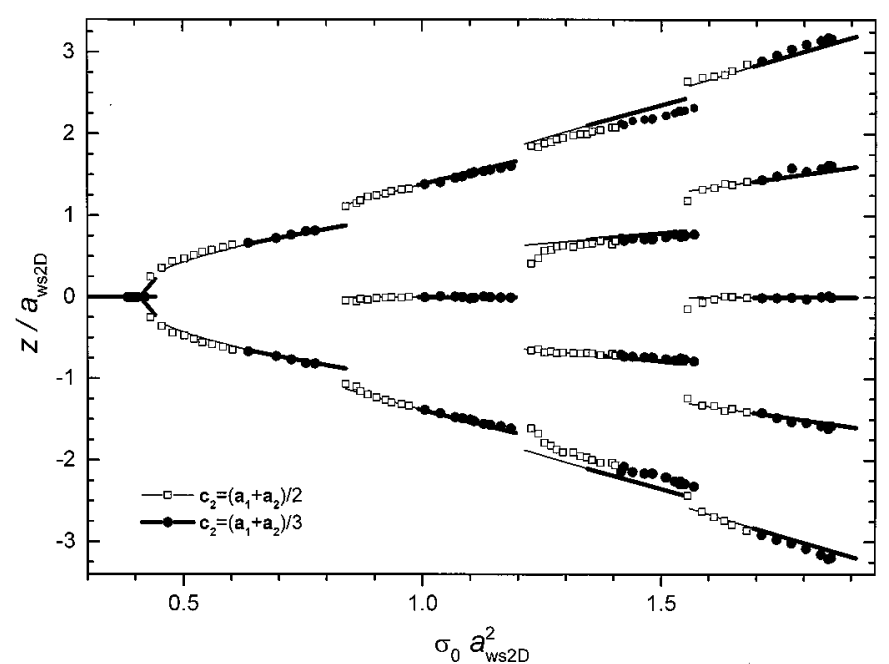

Fig. 3 (left). Interlayer structure (plane axial positions and displacement vectors) of the central region as a function of normalized central areal charge density. The lines show predictions from theory, and symbols show experimental measurements. The symbols indicate whether the lattices had an interlattice displacement vector $c_{2}$ that was characteristic of the hexagonal phases (circles) or the square and rhombic phases (squares). Lengths have been normalized by $a_{\text {WS2D }}=\left(3 e^{2} / 4 \pi \epsilon_{0} m \omega_{z}^{2}\right)^{1 / 3}$

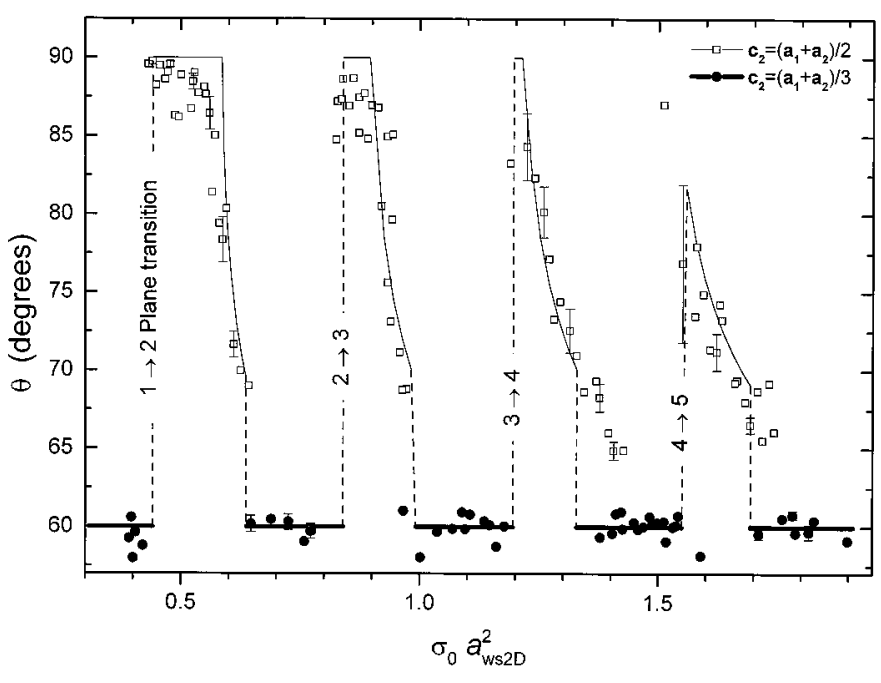

$=10.7 \mu \mathrm{m}$, which is the Wigner-Seitz radius in the planar limit. Fig. 4 (right). Intralayer angle $\theta$ of the central region as a function of central areal charge density. The solid lines indicate the minimum energy structures that are predicted by the 2D theory. The symbols indicate experimental measurements, which are from the same data sets that were used in Fig. 3. Some representative error bars that indicate the scatter in the measurements are included. $\phi_{e}=1 / 2(m / e) \omega_{z}^{2} z^{2}$. Because this potential is identical to the confinement potential of a Penning trap in the $\alpha \rightarrow 0$ planar limit (26), this theory should predict the structures that were observed in the central region of the lenticular plasmas of the experiments. The predictions of this two-dimensional (2D) theory, which has no free parameters, were compared directly with our observations by identifying the areal density of the planar OCP with the directly measured central areal density $\sigma_{0}$ of the lenticular plasmas. For a quantitative analysis of the observed lattice structure, we performed a least squares fit of the positions of the ions in the central region to the relevant phases (shown in Table 1) (lines in Fig. 2). Using the best-fit values of the primitive vector length $a$ and the intralayer angle $\theta$ and using the observed number of lattice planes $n$, we calculated the central areal density $\sigma_{0}$ $=n /\left(a^{2} \sin \theta\right)$.

The agreement between the planar OCP theory and experiment, with measurements taken on different plasmas with $N<10^{4}$, was good (Figs. 3 and 4). As the central areal density was increased, the lattice planes moved farther apart axially (Fig. 3). Eventually, it became energetically favorable to form an additional lattice plane. However, although the phase $\mathrm{V}_{\mathrm{fcc}}$ was predicted to be slightly more energetically favorable than phase $\mathrm{V}$, we rarely observed $\mathrm{V}_{\text {fcc }}(\sim 5 \%$ of the time). These and other minor discrepancies from theory may be due to the finite radial extent of the ion plasma; we observed a similar preference for hcp stacking in molecular dynamics simulations of small $(N=$ $3000)$ lenticular ion plasmas.
For the dependence of the angle $\theta$ (between the primitive vectors $\mathbf{a}_{1}$ and $\mathbf{a}_{2}$ ) on central areal density $\sigma_{0}$ (Fig. 4), the general trend is that, when a new lattice plane is formed, $\theta$ changes discontinuously from $\sim 60^{\circ}$ to a higher value. As $\sigma_{0}$ of the crystal was further increased, $\theta$ smoothly decreased to $\sim 65^{\circ}$ until there was a second discontinuous transition to a hexagonal structure. This second transition has been predicted to become continuous, with $\theta$ assuming all values $60^{\circ} \leq \theta \leq 90^{\circ}$, in liquid $(\Gamma<80)$ bilayer systems (27). At central areal charge densities near phase boundaries, both phases can be observed. In these regions, the phase that materializes after the crystal is formed is initially random but tends to persist if the ions are not heated. Where there was no strong preference for one phase over the other, both were plotted (Fig. 4).

Like most materials, the hexagonal and square phases contract in lateral directions when elongated. However, the rhombic phase shows quite different behavior because the intralayer angle $\theta$ strongly depends on the $\hat{\mathbf{z}}$-axis strain; one rhombus diagonal contracts, and the second expands when the rhombic phase is elongated in the $\hat{\mathbf{z}}$-axis direction. The dimensional change for the latter diagonal corresponds to a negative value of the Poisson's ratio (which is the ratio of the lateral contraction to the longitudinal elongation). The present experimental observations substantially expand the mass density range over which negative Poisson's ratios have been established [from $\sim 10 \mathrm{~g} / \mathrm{cm}^{3}$ for cubic metals (28) and $\sim 0.1 \mathrm{~g} / \mathrm{cm}^{3}$ for reentrant foams (29) to $\sim 10^{-15} \mathrm{~g} / \mathrm{cm}^{3}$ for the present ion crystals].
References and Notes

1. H. M. Van Horn, Science 252, 384 (1991).

2. C. C. Grimes and G. Adams, Phys. Rev. Lett. 42, 795 (1979).

3. D. J. Wineland, R. E. Drullinger, F. L. Walls, ibid. 40, 1639 (1978).

4. J. N. Tan, J. J. Bollinger, B. M. Jelenković, D. J. Wineland, ibid. 72, 4198 (1995); W. M. Itano et al., Science 279, 686 (1998)

5. I. Waki, S. Kassner, G. Birkl, H. Walther, Phys. Rev. Lett. 68, 2007 (1992); G. Birkl, S. Kassner, H. Walther, Nature 357, 310 (1992)

6. P. K. Ghosh, Ion Traps (Clarendon, Oxford, 1995).

7. C. W. Roberson and C. F. Driscoll, Eds., Non-Neutral Plasma Physics (American Institute of Physics, New York, 1988).

8. S. Ichimaru, H. Iyetomi, S. Tanaka, Phys. Rep. 149, 91 (1987).

9. D. H. E. Dubin, Phys. Rev. E 42, 4972 (1990).

10. J. P. Schiffer, Phys. Rev. Lett. 70, 818 (1993); D. H. E. Dubin, ibid. 71, 2753 (1993).

11. G. Goldoni and F. M. Peeters, Phys. Rev. B 53, 4591 (1996).

12. J. B. Pieper, J. Goree, R. A. Quinn, J. Vac. Sci. Technol. A 14, 519 (1996); H. Totsuji, T. Kishimoto, C. Totsuji, Phys. Rev. Lett. 78, 3113 (1997).

13. D. H. Van Winkle and C. A. Murray, Phys. Rev. A 34, 562 (1986).

14. S. Narasimhan and T.-L. Ho, Phys. Rev. B 52, 12291 (1995); G. Goldoni and F. M. Peeters, Europhys. Lett. 37, 293 (1997).

15. M. Schmidt and H. Löwen, Phys. Rev. E 55, 7228 (1997).

16. R. Rafac, J. P. Schiffer, J. S. Hangst, D. H. E. Dubin, D. J. Wales, Proc. Natl. Acad. Sci. U.S.A. 88, 483 (1991).

17. F. Diedrich, E. Peik, J. M. Chen, W. Quint, H. Walther, Phys. Rev. Lett. 59, 2931 (1987); D. J. Wineland, J. C. Bergquist, W. M. Itano, J. J. Bollinger, C. H. Manney, ibid., p. 2935.

18. A. Rahman and J. P. Schiffer, ibid. 57, 1133 (1986); D. H. E. Dubin and T. M. O'Neil, ibid. 60, 511 (1988)

19. S. L. Gilbert, J. J. Bollinger, D. J. Wineland, ibid., p. 2022.

20. M. Drewsen, C. Brodersen, L. Hornekaer, J. S. Hangst, J. P. Schiffer, ibid. 81, 2878 (1998).

21. X.-P. Huang, J. J. Bollinger, T. B. Mitchell, W. M. Itano, ibid. 80, 73 (1998); Phys. Plasmas 5, 1656 (1998).

22. L. R. Brewer et al., Phys. Rev. A 38, 859 (1988).

23. T. M. O'Neil, Phys. Fluids 24, 1447 (1981); D. J. Larson et al., Phys. Rev. Lett. 57, 70 (1986). 
24. T. B. Mitchell, J. J. Bollinger, D. H. E. Dubin, X.-P. Huang, W. M. Itano, in preparation (preprint available at www.boulder.nist.gov/timefreq/ion/penning/pop/ pop.pdf).

25. Minimization of the energy was carried out numerically for given areal density by allowing the positions of the planes to vary with respect to one another within the external potential and by varying the lattice structure within the planes. We made the simplifying assumption, which was consistent with the experimental observations, that the lattice structure of each plane was identical. The areal density of the planar OCP is a control parameter that, when varied, leads to transitions in both the number and lattice structure of the planes. This theory focuses on the minimum potential energy states while neglecting finite temperature effects that may be important (particularly effects that are near the continuous phase transitions where large structural fluctuations may occur).

26. T. M. O'Neil and D. H. E. Dubin, Phys. Plasmas 5, 2163 (1998).

27. V. I. Valtchinov, G. Kalman, K. B. Blagoev, Phys. Rev. E 56, 4351 (1997).
28. R. H. Baughman, J. M. Shacklette, A. A. Zakhidov, S. Stafstrom, Nature 392, 362 (1998).

29. R. Lakes, Science 235, 1038 (1987).

30. This manuscript is a work of the U.S. government; it is not subject to U.S. copyright. This research was supported by the Office of Naval Research and NSF (D.H.E.D.). We thank B. King, R. J. Rafac, J. P. Schiffer, and D. J. Wineland for useful comments and B. M. Jelenković and J. N. Tan for technical assistance.

14 August 1998; accepted 14 October 1998

\section{Controlling Factors for the Brittle-to-Ductile Transition in Tungsten Single Crystals}

\author{
Peter Gumbsch,* Joachim Riedle, Alexander Hartmaier, \\ Hellmut F. Fischmeister
}

\begin{abstract}
Materials performance in structural applications is often restricted by a transition from ductile response to brittle fracture with decreasing temperature. This transition is currently viewed as being controlled either by dislocation mobility or by the nucleation of dislocations. Fracture experiments on tungsten single crystals reported here provide evidence for the importance of dislocation nucleation for the fracture toughness in the semibrittle regime. However, it is shown that the transition itself, in general, is controlled by dislocation mobility rather than by nucleation.
\end{abstract}

Some materials are brittle and shatter like glass, whereas others are ductile and deformable. Several materials, such as the refractory metals, steels, and semiconductor crystals, exhibit both types of behavior (1-5) with a brittle-to-ductile transition (BDT) at a characteristic temperature. A crack introduced into a material may propagate as a brittle crack with an atomically sharp crack front; alternatively, the material near the crack tip may show sufficient plasticity to slow down or arrest the crack. Crack-tip plasticity comprises two distinct processes, nucleation of dislocations at or near the crack tip and their propagation away from the crack. Several models describe the BDT as a nucleation-controlled event $(6,7)$, and others focus on dislocation mobility as the controlling factor $(8-10)$.

The experimental evidence on the controlling factors of the BDT, which has been obtained mostly from silicon single crystals, remains inconclusive. On the one hand, the temperature at which the BDT occurs [BDT temperature (BDTT)] is strongly dependent on the strain rate, which allows an activation energy for the BDT to be determined. This activation energy has been shown to be equal to that for dislocation motion $(2,8,9)$, which suggests a mobility-controlled BDT. On the other hand, specimen size and the availability

Max-Planck-Institut für Metallforschung, Seestrasse 92, 70174 Stuttgart, Germany.

*To whom correspondence should be addressed. Email: gumbsch@finix.mpi-stuttgart.mpg.de of dislocation sources have a pronounced influence on the fracture toughness to the degree that they may even switch the material's behavior from brittle to ductile (11).

We performed cleavage experiments on tungsten single crystals to determine the controlling factors of the BDT. To exclude disturbances from grain boundaries, single crystals were chosen. Fracture toughness tests were performed on all four low-index crack systems. A crack system is specified by the nominal crack plane and the crack front direction. The four crack systems studied are therefore denoted as $\{100\}<010>,\{100\}<011>,\{110\}<1 \overline{1} 0>$, and $\{110\}<001>$ in crystallographic notation. The temperature range covered by the experiments was between the liquid nitrogen temperature $(77 \mathrm{~K})$ and $650 \mathrm{~K}$. The toughness tests were performed on high-purity (12) tungsten single crystal bars ( $3 \mathrm{~mm}$ by $6 \mathrm{~mm}$ by $30 \mathrm{~mm}$ ) loaded in three-point bending. To obtain a welldefined value for the fracture toughness, a sharp crack front was introduced by precracking at $77 \mathrm{~K}$. Precrack length was evaluated from the compliance and, where possible, was also determined postmortem on the fracture surfaces, where faint arrest lines are visible. The tests were performed with a constant loading rate, which translates into an almost constant stress intensity rate of $0.10 \pm 0.02 \mathrm{MPa} \mathrm{m} \mathrm{m}^{1 / 2} / \mathrm{s}$ (13). More details of the experimental procedure are given in (14).

Multiple tests (more than five) were conducted for all four crack systems at room temperature and at $77 \mathrm{~K}$. Several individual tests at other temperatures were performed to determine the temperature dependence of the fracture toughness (Fig. 1). Macroscopically, the transition from brittle to ductile response usually correlates with the maximum in fracture toughness. The temperature at this maximum was taken as the BDTT and was determined from Fig. 1 within 30 to $60 \mathrm{~K}$ (Table 1). The so-defined BDTTs fell into an interval of $100 \mathrm{~K}$ for all four crack systems, even though both $\{110\}$ crack systems had a significantly higher room temperature fracture toughness than the $\{100\}$ systems (Table 1).

Testing at $77 \mathrm{~K}$ always resulted in brittle cleavage fracture on the precrack plane for all four crack systems. The load-displacement curves showed perfectly linear behavior up to the load at which fracture occurred. The fracture surfaces were clean except for a few river lines.

At room temperature and above, most of the fracture specimens revealed small deviations from linearity in the load-displacement curves before final fracture, indicating a limited amount of plastic relaxation. The fracture surfaces were significantly rougher than those of the precracks and of the specimens tested at $77 \mathrm{~K}$. Cleavage below the BDTT was always catastrophic. No noticeable amount of slow crack growth was observed.

The preexisting dislocation density and the availability of dislocation sources can be increased by plastic deformation before toughness testing. Specimens with a $\{110\}<1 \overline{1} 0>$ crack system were deformed by compression along the $\langle 110\rangle$ (long) axis at $400^{\circ} \mathrm{C}$ to a plastic strain of $\varepsilon_{\mathrm{pl}}=0.1$ before precracking. At low temperatures the predeformed specimens are significantly tougher than the unde-

Table 1. BDTT and fracture toughness of tungsten single crystals for the $\{100\}$ and $\{110\}$ cleavage planes with different crack front directions. Fracture toughness at room temperature $K^{\mathrm{RT}}$ and at liquid nitrogen temperature $K^{77 \mathrm{~K}}$ are mean values from at least five individual measurements (standard deviation in parentheses). Fracture toughness is in $\mathrm{MPa} \mathrm{m}^{1 / 2}$.

\begin{tabular}{lcrc}
\hline Crack system & BDTT $(K)$ & \multicolumn{1}{c}{$K^{\text {RT }}$} & $K^{77 K}$ \\
\hline$\{100\}<010>$ & 470 & $8.7(2.5)$ & $3.4(0.6)$ \\
$\{100\}<011>$ & 370 & $6.2(1.7)$ & $2.4(0.4)$ \\
$\{110\}<001>$ & 430 & $20.2(5.5)$ & $3.8(0.4)$ \\
$\{110\}<110>$ & 370 & $12.9(2.1)$ & $2.8(0.2)$ \\
\hline
\end{tabular}

